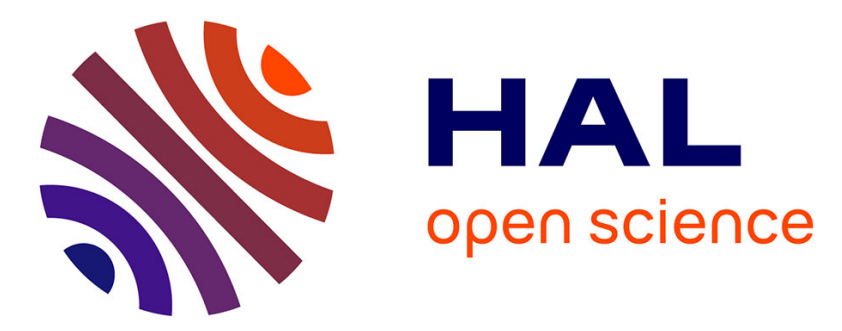

\title{
A Morphological Approach for Vessel Segmentation in Eye Fundus Images, with Quantitative Evaluation
}

Florence Rossant, Maddalena Badellino, Alexandre Chavillon, Isabelle Bloch, Michel Pâques

\section{- To cite this version:}

Florence Rossant, Maddalena Badellino, Alexandre Chavillon, Isabelle Bloch, Michel Pâques. A Morphological Approach for Vessel Segmentation in Eye Fundus Images, with Quantitative Evaluation. Journal of Medical Imaging and Health Informatics, 2011, 1, pp.42 - 49. 10.1166/jmihi.2011.1006 . hal-02559261

\section{HAL Id: hal-02559261 \\ https://hal.science/hal-02559261}

Submitted on 30 Apr 2020

HAL is a multi-disciplinary open access archive for the deposit and dissemination of scientific research documents, whether they are published or not. The documents may come from teaching and research institutions in France or abroad, or from public or private research centers.
L'archive ouverte pluridisciplinaire HAL, est destinée au dépôt et à la diffusion de documents scientifiques de niveau recherche, publiés ou non, émanant des établissements d'enseignement et de recherche français ou étrangers, des laboratoires publics ou privés. 


\title{
A Morphological Approach for Vessel Segmentation in Eye Fundus Images, with Quantitative Evaluation
}

\author{
Florence Rossant ${ }^{2, *}$, Maddalena Badellino ${ }^{1}$, Alexandre Chavillon ${ }^{2}$, \\ Isabelle Bloch ${ }^{1}$, and Michel Paques ${ }^{3}$ \\ ${ }^{1}$ Telecom ParisTech, CNRS LTCI, Paris 75013, France \\ ${ }^{2}$ Institut Supérieur d'Electronique de Paris (ISEP), Paris 75006, France \\ ${ }^{3}$ CIC 503 du CHNO des 1520, INSERM \& Direction de l'Hospitalisation et des Soins, Paris 75011, France
}

\begin{abstract}
In this paper, we propose a method based on morphological and topological analysis for the segmentation of vessels in eye fundus images. This is a very important problem, in particular for the quantitative assessment of microvascular damage due to arterial hypertension, diabetes and aging, such as branch retinal vein occlusions. This paper addresses the segmentation step required for such studies. A pre-processing step includes a morphological filtering to enhance the vessels. Attribute images are then built from a combination of a top-hat transform (i.e., a non linear operation) with linear filters at two different scales, leading to complementary information. Linear structure extraction is then performed using path-opening filters. The final segmentation relies on a fusion step and automatic thresholding. From this segmentation, a graph representation is then extracted, suitable for further quantitative analysis. The method has been evaluated on a large database of images, and good results have been obtained, in particular in terms of accuracy (average of $94.33 \%$ over the whole database, with a standard deviation of 0.61 ) and specificity (average of $97.88 \%$ over the whole database, with a standard deviation of 0.57 ). These results compare favorably to the ones obtained by other methods on the same database. The proposed approach can therefore be further exploited for temporal analysis of retinal diseases.
\end{abstract}

Keywords: Eye Fundus Images, Vessel Segmentation, Mathematical Morphology.

\section{INTRODUCTION}

In the last years, retinal image analysis became a popular research field, three main factors explaining this trend:

1. retina is the only location where blood vessels can be visualized non-invasively in vivo;

2. retinal images can be produced and distributed with low time and financial costs;

3. retinal vessels are strong indicators for the presence of frequent diseases, such as diabetic retinopathy, retinal vessel occlusion, arterial hypertension.

Moreover, in the context of clinical research, methods for automatically analyzing retinal images have a high relevance since they offer the potential to examine a large number of images with time and cost savings and offer more objective measurements than current observer-driven techniques.

Aging leads to increased thickness of the wall of large (atherosclerosis) and/or of small arteries (arteriolosclerosis). While the consequences of the latter on transversal geometry

*Author to whom correspondence should be addressed. (e.g., focal or generalized reduction of lumen, arteriovenous nicking) has been the subject of many investigations, its consequences on longitudinal geometry (e.g., vessel length) are poorly known. It is likely that increased thickness of the arterial wall will increase its length and subsequently its tortuosity. Thus, analysis of age-related changes in retinal vascular anatomy may provide cues about vascular aging, a major cause of diseases of the brain and heart. Furthermore, such age-related changes in arterial pathways may participate to a retinal disease called branch retinal vein occlusions (BRVO) due to the geometrical constraints at arteriovenous crossings, that is, local compression of a vein by a bypassing arteriole.

In order to better understand normal and pathological aging of retinal vessels, the Clinical Investigation Center of the QuinzeVingts hospital in Paris needs dedicated image processing tools, allowing the automatic analysis of eye fundus images and especially the detection of small variations of the geometry of vessels. Our ultimate goal is to quantify the changes in the geometry of retinal vessels over time in the same subject. In this paper we propose, as a first step towards this aim, a method for extracting the vascular tree and then detect the characteristic points of this 
structure (intersection and bifurcation points), based on morphological and topological analysis of the data, and which overcome some of the limitations of existing methods. A discussion on the state of the art highlighting these limitations is given in Section 2.

The proposed segmentation is detailed in Section 3, adapting and extending the approach developed in Refs. [35, 36]. The vascular tree construction and key points identification are also presented in this section. The segmentation method has been evaluated on a large database of images, and results are summarized in Section 4. A discussion and concluding remarks are finally provided in Section 5.

\section{RELATED WORK}

A number of algorithms have been presented in the literature since the last two decades for the segmentation of retinal blood vessels. Many of them are based on the assumption that the intensity profile of the vessels is Gaussian-like. Thus, directional Gaussian filters, $5,6,14,22$ as well as their first or second order derivatives, ${ }^{8,10,22,34,36}$ have been widely applied in order to enhance the blood vessels.

Many other filters have been proposed for highlighting the vessels: morphological filters with linear structuring elements, ${ }^{35,36}$ modified top-hat, ${ }^{21}$ tramline algorithms, ${ }^{2,17}$ line detectors, ${ }^{23}$ wavelets. ${ }^{28}$ In Refs. $[19,29]$, the maximum curvature of the vessels is found by computing the eigenvalues of the Hessian matrix, with the aim of finding the vessel ridges. Recently, Lam proposed three concavity measures extracted from two regularized images, allowing a better discrimination between vessels and retinal lesions. ${ }^{16}$

One key-point is the choice of the matched filter parameters. For example, many authors choose a fixed standard deviation value for the Gaussian model (e.g., Refs. [5, 6, 14, 19, 36]), assuming implicitly that the vessel caliber is invariant inside the FOV area, which is obviously not the case, as underlined in Refs. [3, 10, 11, 22]. The same issue occurs with line detectors and with morphological approaches: in Ref. [21] for example, the size of the structuring element defines four analysis scales. Oppositely, wavelets decomposition allows naturally a multi-scale analysis.

The vessel segmentation relies then on a classification step, which can be either non-supervised or supervised. In unsupervised approaches, the pixels of the enhanced image are classified as vessel or non-vessel, by comparing their intensity level to a threshold. This one can be set for the whole image (e.g., Refs. [5, 6]), calculated locally ${ }^{3}$ for dealing with the intensity and contrast variations throughout the FOV area, or even set according to the filter parameters. ${ }^{10,34}$ Sometimes, several enhanced images are combined, using a probabilistic ${ }^{16}$ or a fuzzy $^{32}$ formalism to achieve the fusion. Due to the difficulty of classifying each pixel without any structural information, Hoover proposed an iterative process, called threshold probing, in which the threshold value used in a region growing process is optimized, based on tests realized on the detected vessel region. ${ }^{14}$ Mendonca combined region growing with binary masks obtained by morphological operations at four different scales. ${ }^{21} \mathrm{~A}$ general framework for "intelligent" thresholding was also proposed by Jiang, and applied on the brute retinal images: the image is probed with a number of thresholds, and, for each one, the binary extracted regions are classified so that only those having vessellike features are retained. ${ }^{15}$
In order to improve the segmentation accuracy, supervised classification schemes have been developed, based on knn-classifiers, ${ }^{22,29}$ neural networks, ${ }^{27} \mathrm{SVM}^{23}$ or Bayesian classifier. ${ }^{28}$ Again, the feature vector, calculated for each pixel, contains intensity values extracted from the vessel enhancing step, $22,23,27,28$ or higher-level knowledge provided by the sub-region including the pixel. ${ }^{29}$ The supervised-classification approaches reach better accuracy than the unsupervised ones. ${ }^{28}$ However, the learning stage requires many manual segmentations, which are long and tedious to realize and have to be redone when dealing with another kind of retina database.

Tracking procedures (e.g., Refs. [2, 4, 7-9, 11, 31]) are also worth mentioning. They range from simple contour tracking under parallelism constraints ${ }^{7,9}$ to more sophisticated approaches relying on vessel profile models. For example, Al-diri used a model derived from the ROT active contour, ${ }^{2}$ Chutatape proposed a Kalman filter incorporating information about vessel width and direction. ${ }^{8}$ The main advantages of tracking procedures are, first, that the computational cost is reduced, ${ }^{4}$ since only a part of the image is explored, and, second, that the method leads to a connected vascular network. However, the tracking is always realized based on local properties and bifurcation points are difficult to handle. So there is a real risk of error. Especially, if the tracking stops prematurely, an important part of the vessel tree may be definitely lost.

Finally, advanced active contour models, with energy terms integrating parallelism constraints, have been proposed. ${ }^{1}$ However, this approach requires additional steps to detect bifurcation and crossing area. This problem is avoided in Ref. [20] since the ACID framework allows a powerful snake re-parameterization, so that complex structures and topology changes can be handled. But this time, the model may be insufficiently robust, since it does not make use of a priori knowledge.

Based on our literature review, morphological approaches combined with curvature estimation seemed very interesting to us, since they combine structural information, related to the curvilinear shape of the vessel, with intensity profile model, revealed by the Laplacian operator. Moreover, a non-supervised method is absolutely required since we have to process images acquired by different devices, which would lead us to repeat tedious learning procedures. So, our aim is to adapt and extend the method proposed in Refs. [35, 36], with the specific objective of detecting accurately the main vessels (the small ones are not important in our clinical context) and improve the connectivity of the obtained vascular tree.

\section{METHOD: VESSEL SEGMENTATION}

Figure 1 depicts the flowchart of the proposed segmentation method. From the original color images of the eye fundus, the green channel is first extracted, resized and denoised. Then, the preprocessed image is passed to the analysis process, consisting of two main stages:

- Filtering, allowing highlighting the vessel features and extracting two attribute images, in which the vessels appear more contrasted.

- Path-opening, applied to each attribute image so that the main elongated features are preserved whereas the other ones are suppressed. 


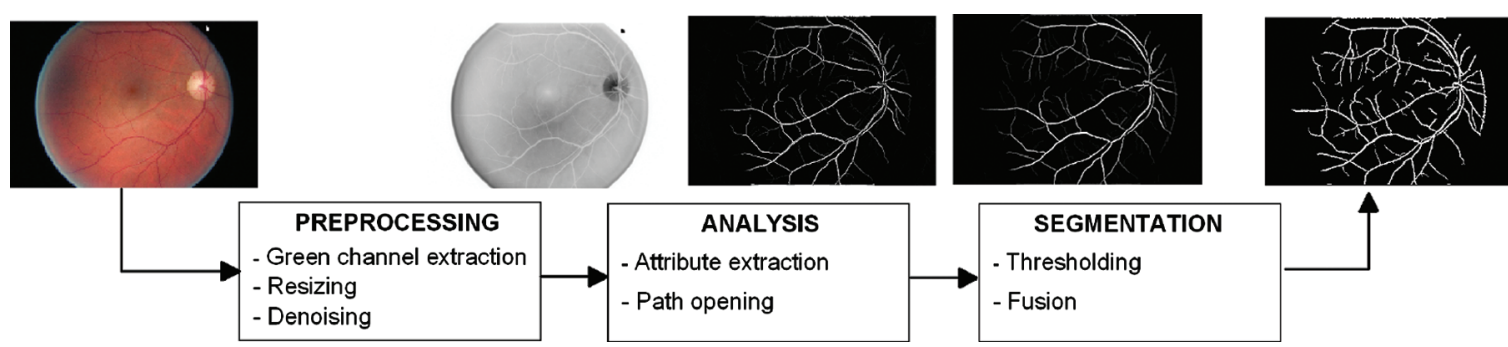

Fig. 1. Flowchart of the proposed segmentation method.

- Finally, the two resulting images are binarized and fused, to provide the final segmentation image where vessel pixels are labeled ' 1 ' and background pixels ' 0 '.

\subsection{Preprocessing}

As the blood vessels appear more contrasted in the green band than in the red or blue ones, only the green channel will be processed. The user is then invited to define the diameter $d_{\max }$ of the thickest vessel, simply by clicking with the mouse on two border pixels. This interactive stage is not very coercive and well accepted by medical experts, and permits to define more accurately the operators used thereafter, this maximal diameter $d_{\max }$ serving as a reference value. Moreover, it can be easily bypassed by just using a standard value. A typical value of $d_{\max }$ is 7 pixels for an eye fundus image of 512 rows.

The image is then preprocessed. A Gaussian filter followed by an opening of size 1 is applied, in order to eliminate the noise and small "walls" that may disconnect the vascular tree ${ }^{35}$ (Fig. 2).

(a)

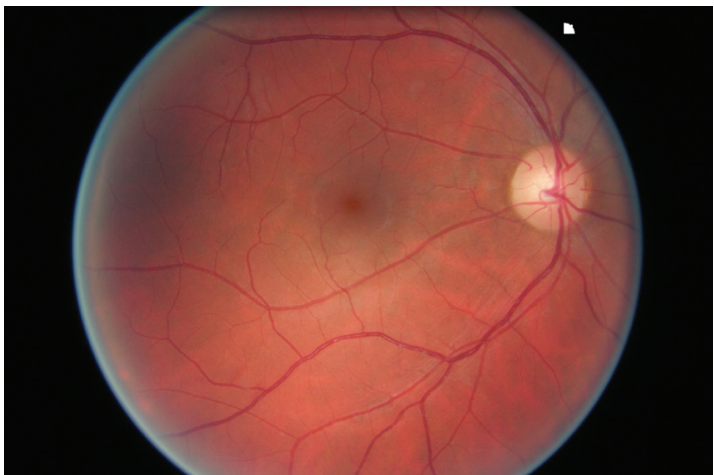

(b)

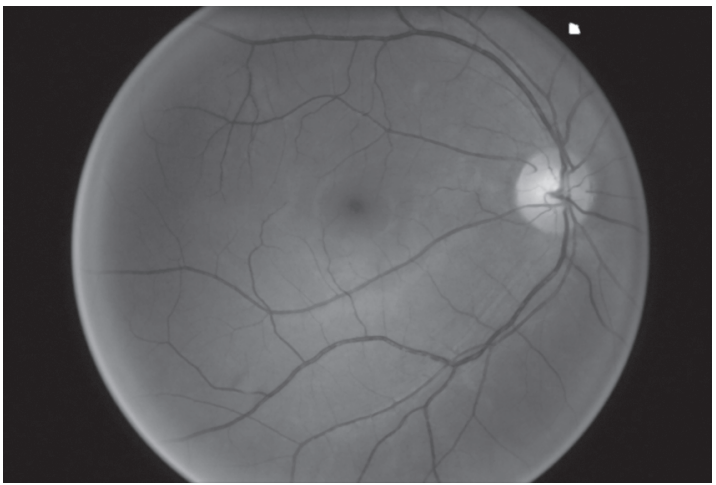

Fig. 2. Original image and preprocessed green channel (image g).
On the resulting image, denoted by $g$, a toggle mapping filter is then applied. ${ }^{26,35}$ This filter is based on the comparison between the input image $g$ and its opening $\gamma$ and closing $\phi$ (see Ref. [25] for the definitions and properties of the morphological operators):

$$
\begin{aligned}
h(x) & =[T M(g)](x) \\
& = \begin{cases}\phi_{B_{1}}(g)(x) & \text { if } \phi_{B_{1}}(g)(x)-g(x) \leq g(x)-\gamma_{B_{2}}(g)(x) \\
\gamma_{B_{2}}(g)(x) & \text { if } \phi_{B_{1}}(g)(x)-g(x)>g(x)-\gamma_{B_{2}}(g)(x)\end{cases}
\end{aligned}
$$

where $\gamma_{B}$ and $\phi_{B}$ denote the opening and the closing with a structuring element $B$, respectively.

In our application, the structuring element $B_{1}$ is a disk whose radius is chosen so that most vessels are completely suppressed in the closing image (Fig. 3). Therefore, the grey-level value $g(x)$ of a vessel pixel $x$ is closer to the opening than to the closing, and so it will be darkened, whereas a background pixel will take the value of the closing. Thus, the contrast of the blood vessels

(a)

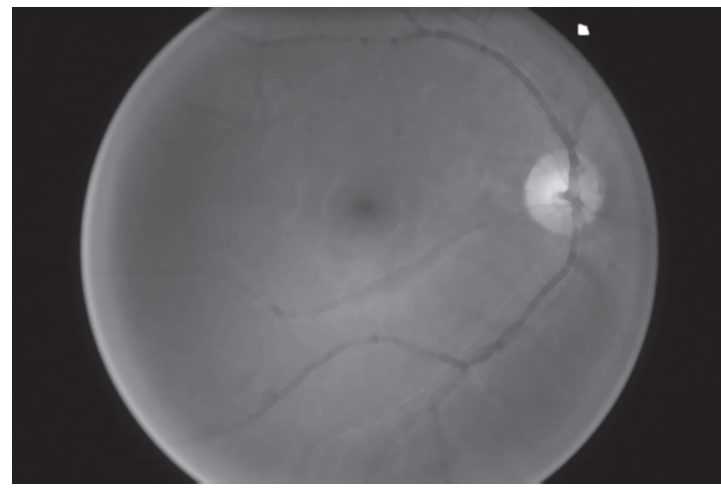

(b)

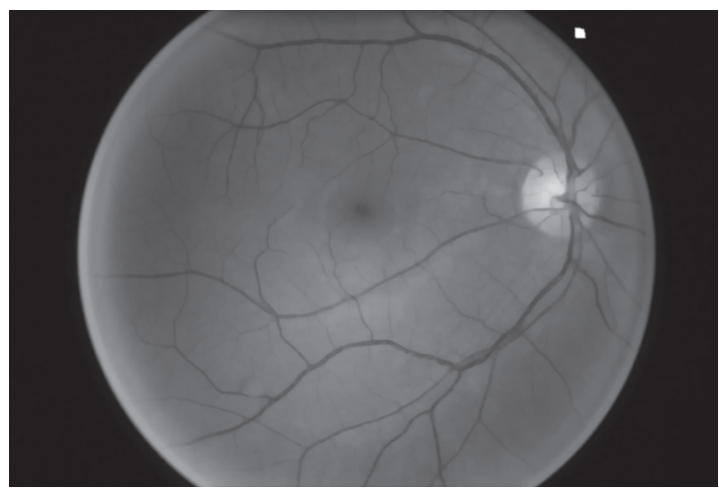

Fig. 3. (a) Closing $\phi_{B_{1}}(g)$ with a disk of size 4. (b) Opening $\gamma_{B_{2}}(g)$ with a disk of size 3 . 
is increased, as explained in Ref. [35]. Especially, some of the bright areas that can be observed in the middle of the thickest arteries are filled. In our experiments we used a disk of size 4 for $B_{1}$ and a disk of size 3 for $B_{2}$ (the same values have been used for all processed cases).

\subsection{Attribute Extraction}

Retinal blood vessels appear as elongated connected structures having approximately a Gaussian profile. ${ }^{36}$ Morphological operations and adapted filtering are applied on the inverted image (Fig. 4) in order to highlight these features.

The top-hat morphological operator is defined as the difference between the original image $h$ and its opening. ${ }^{25}$ By choosing as structuring element a disk whose diameter is slightly larger than $d_{\text {max }}$, all bright features smaller than $d_{\max }$ will be suppressed by the opening while larger features will remain almost unchanged. Thus, the top-hat operation will set to zero the background areas, while the vessels will be enhanced, as shown in Figure 5.

The obtained image $a t t_{0}$ is well contrasted but noisy. Indeed, other small features, smaller than the structuring element, have also been highlighted.

One characteristic of the vessels is their Gaussian-like profile, whose curvature is locally stable along the crest line. Zana and Klein demonstrated in Ref. [36] that the sign of the curvature can be approximated by the sign of the Laplacian. Thus, they proposed to convolve the image with a Gaussian filter and then to compute the Laplacian. The main issue is the choice of the standard deviation of the Gaussian filter, which should be set according to the size of the vessels (matched filter). Most authors choose a single fixed value although all ranges of diameters are possible, from $d_{\max }$ pixels (main vessel near the optical disk) to 1 or two pixels (small capillaries). For this reason, we propose, as an original feature of the method, to process the top-hat image at two different resolutions, by convolving it with the negative of two different Laplacian of Gaussian kernels, $k_{1}$ and $k_{2}$, whose standard deviations are set as follows:

$$
a t t_{i}=a t t_{0} * k_{i}, i=1,2 \text { with } \sigma_{1}=d_{\max } / 6, \sigma_{2}=d_{\max } / 3
$$

The negative values of the results are then set to zero. Figure 6 shows the obtained attribute images. Non-vessel structures are attenuated in both images. With the largest standard deviation $\left(a t t_{2}\right)$, the main veins and arteries are very well detected, without holes and with regular contours. Nevertheless, disconnections

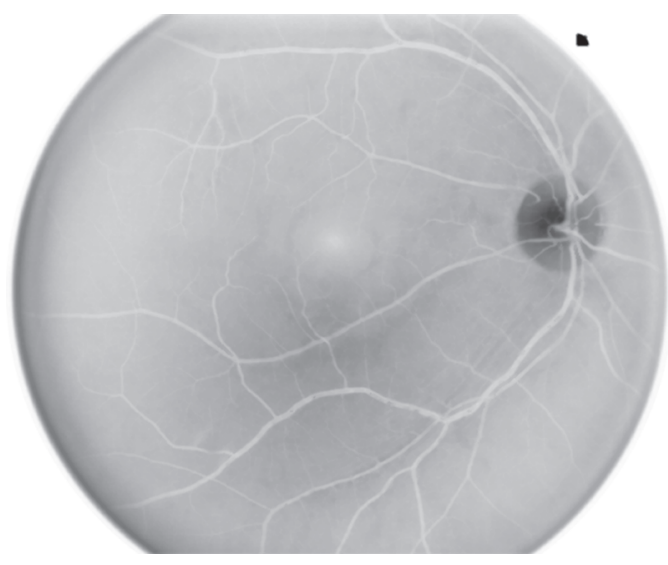

Fig. 4. Input image $h$ of the attribute extraction process.

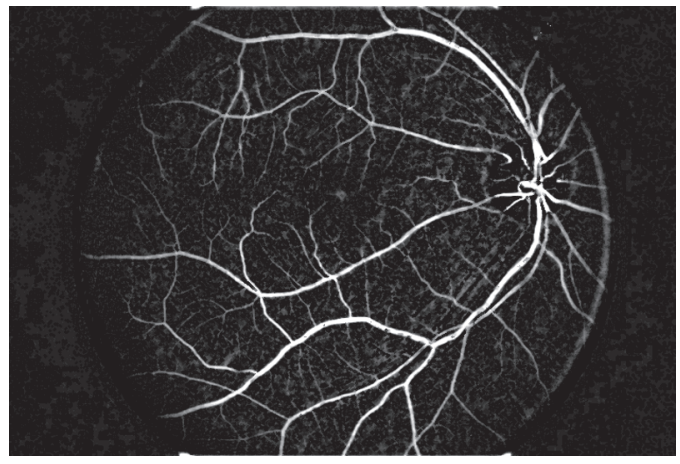

Fig. 5. Top-hat image att 0 .

at bifurcation or crossing areas can be observed and capillaries are not precisely extracted. On the contrary, with the smallest standard deviation $\left(a t t_{1}\right)$ the capillaries are better highlighted and important connections are better preserved. Thus, both attribute images complement each other. The next step aims at improving these results by extracting the main elongated features.

\subsection{Linear Structure Extraction Based on Path-Opening Filters}

Path-opening is an advanced morphological filter which extends the idea of applying openings with straight line segments as structuring elements, in order to detect elongated features. Rather than restricting ourselves to particular directions, we will work with paths that are given by an adjacency relation on the image domain, and our structuring element will be paths of a given length $L .^{12,33}$ This approach is well suited for our

(a)

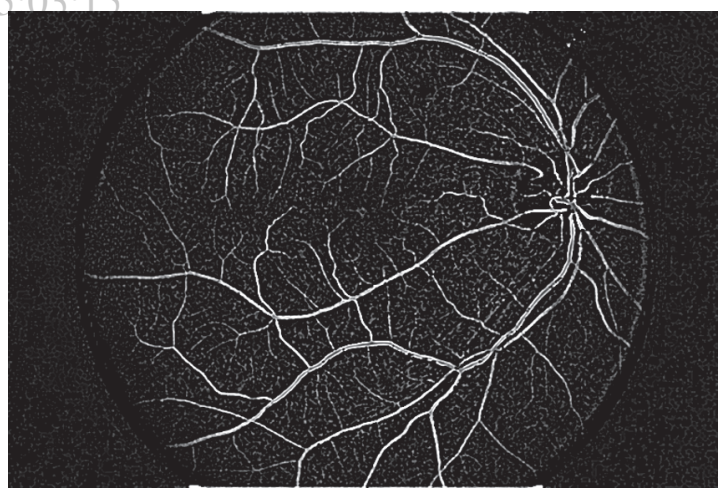

(b)

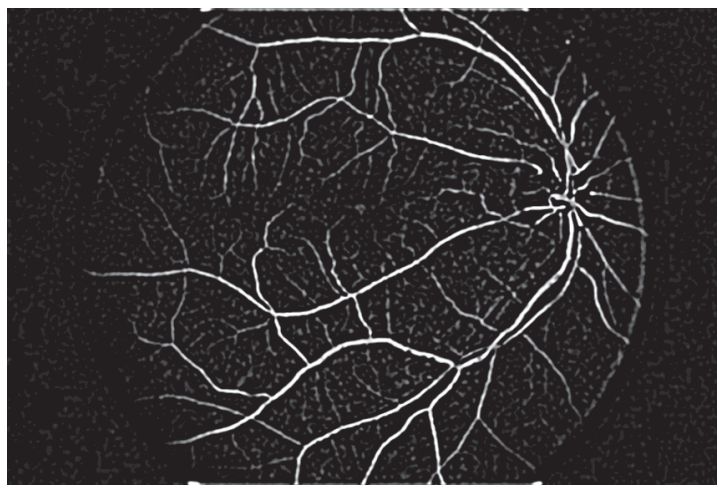

Fig. 6. The two attribute images att ${ }_{1}$ (a) and att ${ }_{2}$ (b) obtained from the top-hat image with $\sigma_{1}$ and $\sigma_{2}$. The images have been calibrated for a better visualization. 
application, since retinal blood vessels are not completely linear, even locally. ${ }^{32}$ The path-opening filter ${ }^{32}$ is applied to both attribute images, allowing retaining elongated bright structures whose length is larger than $\mathrm{L}$ and removing the others. We use $L=40$, which is a good compromise between robustness and computation cost. The implementation relies on the fast algorithm proposed in Ref. [30]. In the following, we denote by $\alpha_{i}$ the images obtained using this filter on $a t t_{i}$ (Fig. 7).

\subsection{Classification and Fusion}

The pixels of each processed attribute image $\alpha_{i}$ are classified into vessel ' 1 ' or background ' 0 ', by hysteresis thresholding. The difficult aspect of this step is the choice of the low and high thresholds, respectively $S_{L}$ and $S_{H}$, which have to be automatically adapted to each case. For that, we propose to define them with respect to an "optimal" threshold $S_{\text {opt }}$, determined via the following ratio:

$$
r(S)=\frac{\# \text { of connected objects }}{\# \text { pixels set to ' } 1 \text { ' }}
$$

Since the vascular tree is completely interconnected, the minimization of $r(S)$ (over admissible values for $S$ ) leads to an "optimal" threshold $S_{\text {opt }}$, for which the number of false detections and non detections is minimal. The low and high thresholds are then defined with respect to $S_{\text {opt }}: S_{H}$ is equal to $S_{\text {opt }}$ while $S_{L}$ is slightly smaller, in order to minimize vessel disconnections at bifurcation or crossing points. More precisely, $S_{L}$ was defined such that $1 \%$ more pixels are assigned to vessels. Figure 8 shows the segmented images, denoted by $\alpha_{i}^{S}$.

A first segmentation result $I_{\text {seg0 }}$ is obtained by the fusion of both segmented images, using the logical OR operator. Then the

(a)

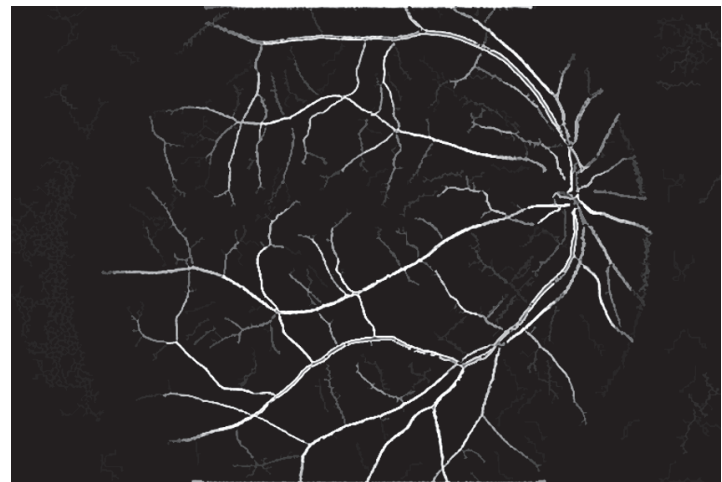

(b)

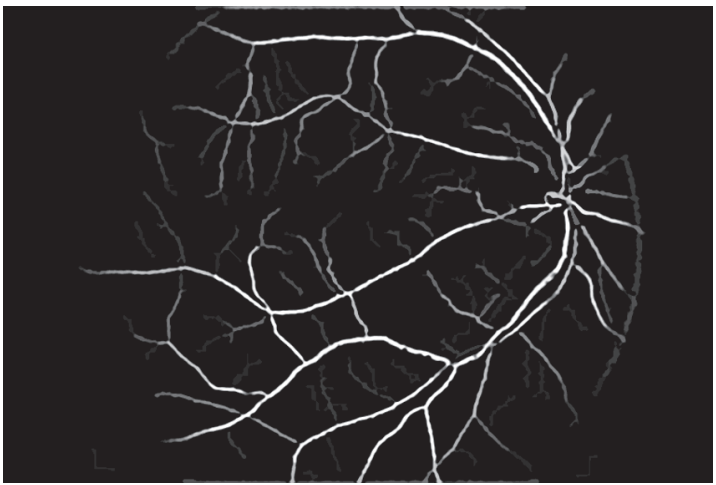

Fig. 7. Attribute images filtered by the morphological path-opening operator $\left(\alpha_{i}\right)$. The images have been calibrated for a better visualization. (a)

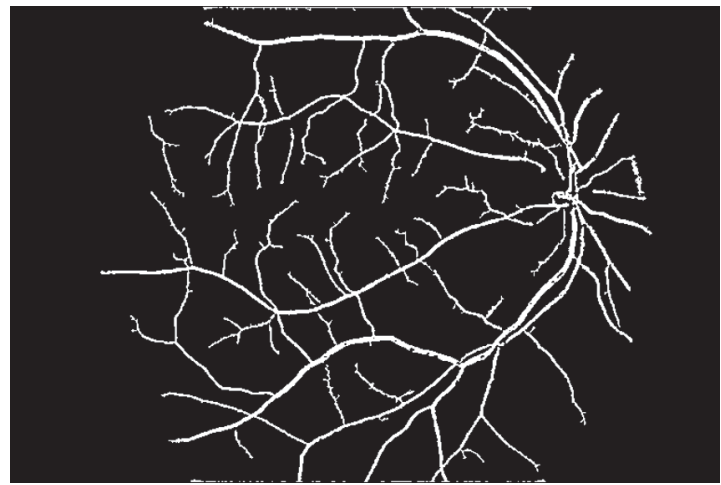

(b)

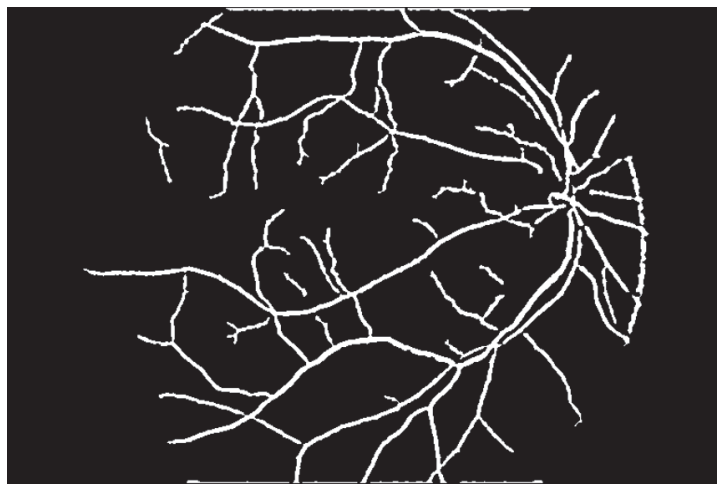

Fig. 8. Segmentation of attribute images (images $\alpha_{i}^{S}$ ).

algorithm proceeds to a step of cleaning. This step follows the idea developed in Ref. [36] where the maximum of openings with linear structuring elements is computed. Note that the result is an algebraic opening. ${ }^{13}$ The final segmentation image $I_{\text {seg }}$ (Fig. 9) is obtained with structuring elements $L_{i}$ of size $1.5 d_{\max }$ representing 12 directions:

$$
I_{\mathrm{seg}}=\max _{i=1, \ldots, 12} \gamma_{L_{i}}\left(I_{\mathrm{seg} 0}\right)
$$

\subsection{Building a Graph Representing the Vascular Tree}

For several applications, such as registration of images acquired for longitudinal studies, it may be convenient to handle a simplified representation of the vessels. Based on the segmentation result, such a representation can be obtained from its skeleton, so as to provide a set of fine lines, representing the vascular tree. Keypoints such as junctions and extremities can then be easily extracted.

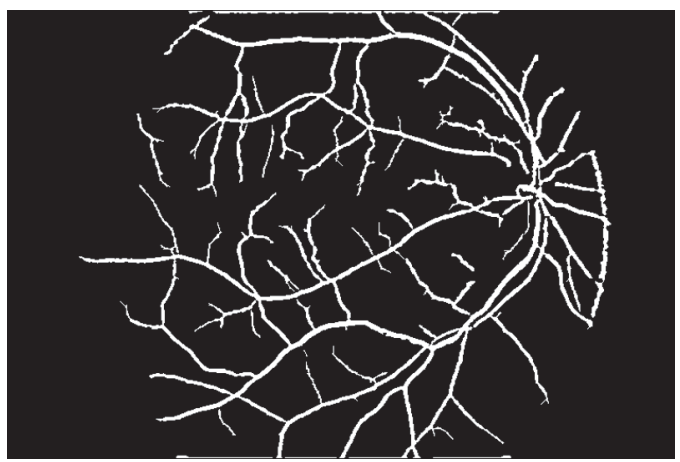

Fig. 9. Final classification image $\left(I_{\text {seg }}\right)$. 


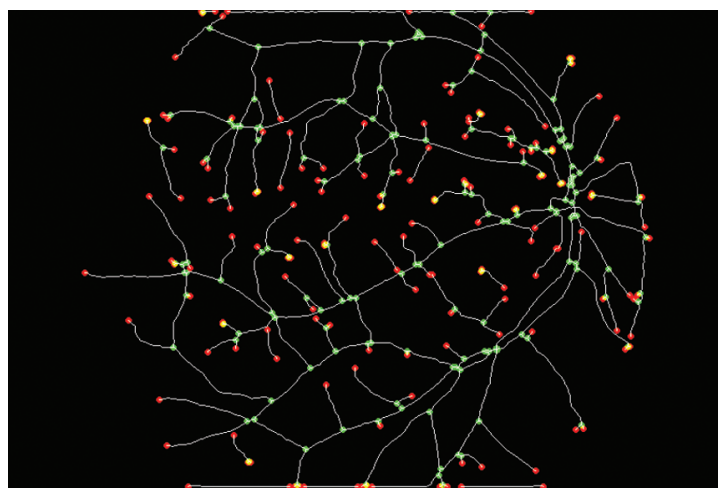

Fig. 10. Skeleton of the segmented vessels, and detection of extremities (red) and junction points (green).

The skeleton is first computed on the segmented image $I_{\text {seg }}$. A thinning-based skeleton is used, so as to preserve the topology. ${ }^{25}$ Since the best connected result is obtained from the binarized top-hat $\alpha_{0}^{S}$ (att 0 processed as $a t t_{1}$ and $a t t_{2}$, according to Sections 3.3 and 3.4), the skeleton is also applied to this image, and segments that were not detected in $I_{\text {seg }}$ are added to the first skeleton if they are connected to it. This allows us on the one hand to obtain a topologically correct skeleton, and on the other hand to have a representation of as many vessel branches as possible.

Topological characteristics of the skeleton points allow us to classify them and to detect end-points (or extremities), and junction points (or bifurcations and crossing points). An extremity has only one skeleton point in its neighborhood, while a junction point has at least three.

An example of result is displayed in Figure 10 and illustrates the final skeleton (after fusion of the branches under connectivity constraint), and the detection of key-points.

The detection of end-points allows in a next step restoring further the connectivity that may be lost during the segmentation. Candidates for reconnection are selected among the end-points based on their distance (here a maximum distance of 10 pixels is used) and the grey levels along the segment joining the end-points. The segment between two end-points with a maximal average grey level (in the original image) along the segment is selected. A result is illustrated in Figure 11, showing the better connectivity obtained after this post-processing step.

Finally, a high-level description of the vascular tree is derived, as a graph, where vertices correspond to the specific points of
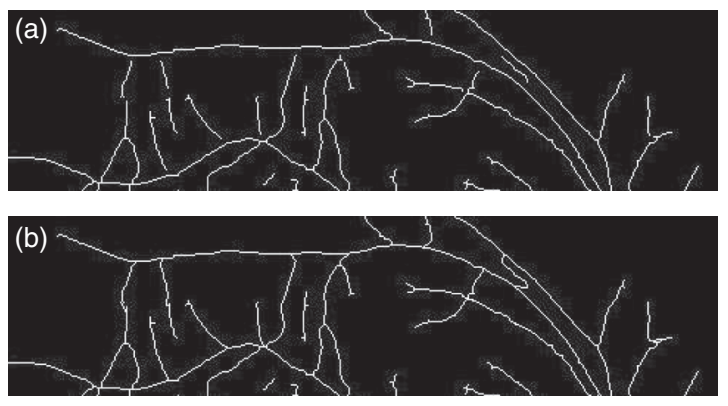

Fig. 11. (a) A subregion of the skeleton of the segmented vessels. (b) The same region after the reconnection procedure.
Table I. Accuracy results for existing methods.

\begin{tabular}{|c|c|c|c|}
\hline Method & Accuracy & Source & Type of method \\
\hline 2nb observer & 0.9473 & Staal $(2004)^{29}$ & Manual \\
\hline Chaudhuri et al. ${ }^{6}$ & 0.8773 & Niemeijer $(2004)^{22}$ & Unsupervised \\
\hline Zana et al. ${ }^{36}$ & 0.9377 & Niemeijer $(2004)^{22}$ & Unsupervised \\
\hline Jiang et al. ${ }^{15}$ & 0.8911 & Staal $(2004)^{29}$ & Unsupervised \\
\hline Rossant et al. & 0.9433 & This paper & Unsupervised \\
\hline $\begin{array}{l}\text { Mendonca green } \\
\text { channel }^{21}\end{array}$ & 0.9452 & Mendonca $(2006)^{21}$ & Unsupervised \\
\hline Line detector ${ }^{23}$ & 0.9563 & Ricci $(2007)^{23}$ & Unsupervised \\
\hline Martinez-Perez et al. ${ }^{18}$ & 0.9181 & Niemeijer $(2004)^{22}$ & Unsupervised \\
\hline $\begin{array}{l}\text { Matched filter knn } \\
(\text { Niemeijer } 04)^{22}\end{array}$ & 0.9416 & Niemeijer $(2004)^{22}$ & Supervised \\
\hline PBM (Staal 2004) ${ }^{29}$ & 0.9441 & Staal $(2004)^{29}$ & Supervised \\
\hline Ricci $(2007)^{23}$ & 0.9595 & Ricci $(2007)^{23}$ & Supervised \\
\hline Soares $(2006)^{28}$ & 0.9466 & Soares $(2006)^{28}$ & Supervised \\
\hline
\end{tabular}

interest, and edges correspond to either vessels or arteries. This structure can then be used in an approach like the one proposed in Ref. [24] Attributes can also be computed, such as vessel thickness and tortuosity, and position of crossings.

\section{RESULTS: SEGMENTATION EVALUATION}

The proposed method has been tested on the DRIVE (Digital Retinal Images for Vessel Extraction) database, ${ }^{a}$ which contains 40 color eye fundus images (with 7 showing a pathology), acquired using a Canon CR5 non-mydriatic 3CCD camera with a 45 degrees field of view (FOV). ${ }^{29}$ The photographs were obtained from a diabetic retinopathy screening program in The Netherlands. Images have been anonymized. Each image is captured using 8 bits per color plane and has a size of $768 \times 584$ pixels. The FOV of each image is circular with a diameter of approximately 540 pixels. Manual segmentations are available and have been performed by three observers. ${ }^{29}$ The database is decomposed in a training set of 20 images and a test set of 20 images.

The proposed method was evaluated by comparing the obtained results to the manual segmentations, using classical measures of accuracy, sensitivity and specificity. Considering the twenty images of the test database, the obtained average measures are as follows: accuracy $=94.33 \%$ (with a standard deviation of 0.61 ), sensitivity $=70.31 \%$ (with a standard deviation of 4.60 ), specificity $=97.88 \%$ (with a standard deviation of 0.57 ). These results are very good, in particular in terms of accuracy and specificity. The somewhat less good sensitivity values are due to the fact that the method focuses on the main vessels only, while the ground truth includes more vessels. These results are among the best reported in the literature for unsupervised methods on the same database, with only $1 \%$ lower accuracy than the best reported result. Noticeably, they are also within the range of the expert results, considering the inter-expert variability $(94.73 \%){ }^{29}$ Table I provides a comparison with other methods.

An example of segmentation is shown in Figure 12. The obtained results are visually good and precise. The network is well connected, even better than reported results on this database, and this is an important feature provided by the proposed method.

\footnotetext{
${ }^{a}$ http://www.isi.uu.nl/Research/Databases/DRIVE.
} 

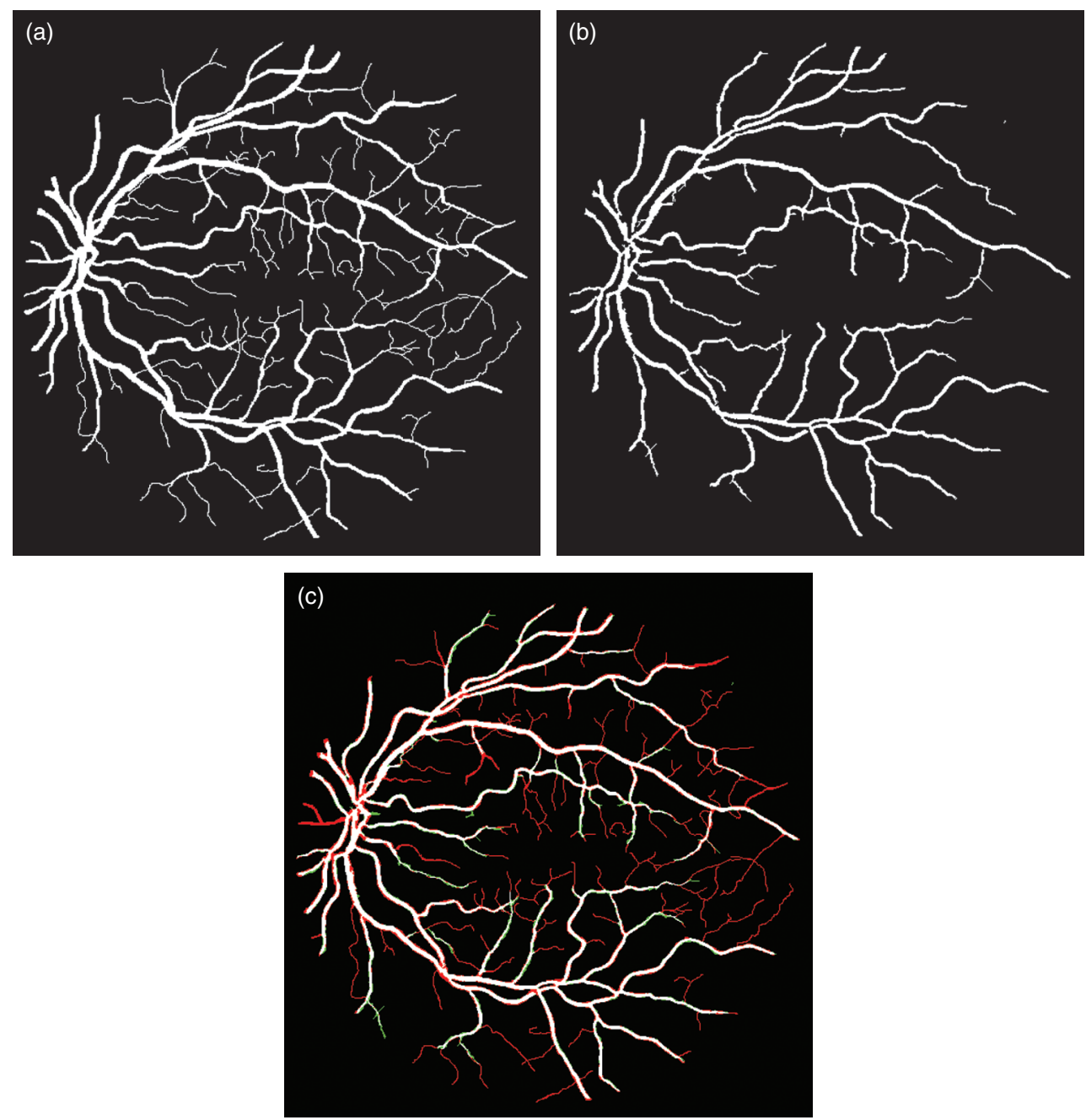

Fig. 12. (a) Manual segmentation. (b) Automated result. (c) Comparison: common parts in white, differences in red (parts from the manual segmentation missed in our result) and green (over-segmentation).

\section{DISCUSSION AND CONCLUSION}

In this paper we proposed an automated method for segmenting the vascular network in eye fundus images. The method improved existing work in particular by making use of two different sets of parameters and combining the results. This allows overcoming several drawbacks of usual methods requiring to make a compromise between connectivity of the vessels and false detections.

Results have been evaluated on a large database including pathological cases. A very good specificity is obtained, and the lower value of sensitivity is due to very narrow vessel extremities which are not taken into account in the proposed approach. This is the main difference with the manual segmentations. Overall, the results remain in the range of the expert segmentations, given their variability. Another important result is that the connectivity of the vessel network is well preserved by the proposed approach, and this is also an improvement over existing methods.

A structured representation is then derived, as an attributed graph, which will be the basis for further analysis. This graph is built from a topologically correct skeleton of the resulting segmentation and from the detection of junction points. These topological features can be correctly extracted thanks to the strong connectivity properties of the segmentation result.

Future work aims at exploiting these results for differentiating between veins and arteries, for registering images acquired at different dates, finding corresponding points in successive images, and for longitudinal follow-up. This will allow us to quantify and model changes over time, in particular changes of tortuosity and shifting of arteriovenous crossings. Correlation to the degree of arteriolosclerosis using gold standards (arteriovenous nicking, arteriovenous ratio, presence of retinopathy) will then be possible.

\section{References and Notes}

1. B. Al-Diri and A. Hunter, A ribbon of twins for extracting vessel boundaries, European Medical and Biological Engineering Conference (EMBEC'05) (2005), pp. 1727-1983.

2. B. Al-Diri, A. Hunter, and D. Steel, An active contour model for segmenting and measuring retinal vessels. IEEE Transactions on Medical Imaging 28, 1488 (2009).

3. W. Cai and A. Chung, Multi-resolution vessel segmentation using normalized cuts in retinal images, Medical Image Computing and Computer-Assisted Intervention-MICCAI 2006, Copenhagen, Danemark (2006), pp. 928-936. 
4. A. Can, H. Shen, J. N. Turner, H. L. Tanenbaum, and B. Roysam, Rapid automated tracing and feature extraction from retinal fundus images using direct exploratory algorithms. IEEE Transactions on Information Technology in Biomedicine 3, 125 (1999).

5. T. Chanwimaluang and G. Fan, An efficient algorithm for extraction of anatomical structures in retinal images, IEEE International Conference on Image Processing (ICIP 2003) (2003), Vol. 1, pp. 1093-1096.

6. S. Chaudhuri, S. Chatterjee, N. Katz, M. Nelson, and M. Goldbaum, Detection of blood vessels in retinal images using two-dimensional matched filters. IEEE Transactions on Medical Imaging 8, 263 (2002).

7. R. Chrástek, M. Wolf, K. Donath, H. Niemann, and G. Michelson, Automated calculation of retinal arteriovenous ratio for detection and monitoring of cerebrovascular disease based on assessment of morphological changes of retinal vascular system, IAPR Workshop on Machine Vison Applications, Nara, Japan (2002), pp. 240-243.

8. O. Chutatape, L. Zheng, and S. M. Krishnan, Retinal blood vessel detection and tracking by matched Gaussian and Kalman filters, 20th Annual International Conference of the IEEE Engineering in Medicine and Biology Society (1998), Vol. 6, pp. 3144-3149.

9. L. Gagnon, M. Lalonde, M. Beaulieu, and M. C. Boucher, Procedure to detect anatomical structures in optical fundus images. SPIE Medical Imaging 4322, 1218 (2001)

10. L. Gang, O. Chutatape, and S. M. Krishnan, Detection and measurement of retinal vessels in fundus images using amplitude modified second-order Gaussian filter. IEEE Transactions on Biomedical Engineering 49, 168 (2002).

11. X. Gao, A. Bharath, A. Stanton, A. Hughes, N. Chapman, and S. Thom, A method of vessel tracking for vessel diameter measurement on retinal images, IEEE International Conference on Image Processing (ICIP 2001) (2001), Vol. 2, pp. 881-884.

12. H. Heijmans, M. Buckley, and $\mathrm{H}$. Talbot, Path openings and closings. Journal of Mathematical Imaging and Vision 22, 107 (2005).

13. H. J. A. M. Heijmans, Morphological Image Operators, Academic Press, Boston (1994).

14. A. D. Hoover, V. Kouznetsova, and M. Goldbaum, Locating blood vessels in retinal images by piecewise threshold probing of a matched filter response. IEEE Transactions on Medical Imaging 19, 203 (2002).

15. $X$. Jiang and $D$. Mo Jon, Adaptive local thresholding by verification-based multithreshold probing with application to vessel detection in retinal images. IEEE Transactions on Pattern Analysis and Machine Intelligence 25, 131 (2003)

16. B. S. Y. Lam, Y. Gao, and A. W. C. Liew, General retinal vessel segmentation using regularization-based multiconcavity modeling. IEEE Transactions on Medical Imaging 29, 1369 (2010).

17. J. Lowell, A. Hunter, D. Steel, A. Basu, R. Ryder, and R. L. Kennedy, Measurement of retinal vessel widths from fundus images based on 2-D modeling. IEEE Transactions on Medical Imaging 23, 1196 (2004).

18. M. Martinez-Perez, A. Hughes, A. Stanton, S. Thom, A. Bharath, and K. Parker, Retinal blood vessel segmentation by means of scale-space analysis and region growing, Medical Image Computing and Computer-Assisted Intervention-MICCAI'99 (1999), pp. 90-97.

19. M. E. Martinez-Perez, A. D. Hughes, A. V. Stanton, S. A. Thom, A. A. Bharath, and K. H. Parker, Segmentation of retinal blood vessels based on the second directional derivative and region growing, IEEE International Conference on Image Processing (ICIP 99) (1999), Vol. 2, pp. 173-176.

20. T. Mclnerney and D. Terzopoulos, T-snakes: Topology adaptive snakes. Medical Image Analysis 4, 73 (2000)

21. A. M. Mendonca and A. Campilho, Segmentation of retinal blood vessels by combining the detection of centerlines and morphological reconstruction. IEEE Transactions on Medical Imaging 25, 1200 (2006).

22. M. Niemeijer, J. Staal, B. van Ginneken, M. Loog, and M. D. Abramoff, Comparative study of retinal vessel segmentation methods on a new publicly available database. SPIE Medical Imaging 5370, 648 (2004).

23. E. Ricci and R. Perfetti, Retinal blood vessel segmentation using line operators and support vector classification. IEEE Transactions on Medical Imaging 26, 1357 (2007)

24. K. Rothaus, $X$. Jiang, and $P$. Rhiem, Separation of the retinal vascular graph in arteries and veins based upon structural knowledge. Image and Vision Computing 27, 864 (2009)

25. J. Serra, Image Analysis and Mathematical Morphology, Academic Press, New York (1982)

26. J. Serra and L. Vincent, An overview of morphological filtering. Circuits, Systems, and Signal Processing 11, 47 (1992)

27. C. Sinthanayothin, J. F. Boyce, H. L. Cook, and T. H. Williamson, Automated localisation of the optic disc, fovea, and retinal blood vessels from digital colour fundus images. British Journal of Ophthalmology 83, 902 (1999).

28. J. V. B. Soares, J. J. G. Leandro, R. M. Cesar, H. F. Jelinek, and M. J. Cree Retinal vessel segmentation using the 2-D Gabor wavelet and supervised classification. IEEE Transactions on Medical Imaging 25, 1214 (2006)

29. J. J. Staal, M. D. Abramoff, M. Niemeijer, M. A. Viergever, and B. van Ginneken, Ridge based vessel segmentation in color images of the retina. IEEE Transactions on Medical Imaging 23, 501 (2004).

30. H. Talbot and B. Appleton, Efficient complete and incomplete path openings and closings. Image and Vision Computing 25, 416 (2007).

31. Y. A. Tolias and S. M. Panas, A fuzzy vessel tracking algorithm for retinal images based on fuzzy clustering. IEEE Transactions on Medical Imaging 17, 263 (1998).

32. S. Valero, J. Chanussot, J. A. Benediktsson, and H. Talbot, Détection automatique du réseau vasculaire rétinien basée sur la morphologie directionnelle et la fusion de décisions, XIle Colloque GRETSI, Dijon, France (2009).

33. S. Valero, J. Chanussot, J. A. Benediktsson, H. Talbot, and B. Waske, Advanced directional mathematical morphology for the detection of the road network in very high resolution remote sensing images. Pattern Recognition Letters 31, 1120 (2010).

34. K. A. Vermeer, F. M. Vos, H. G. Lemij, and A. M. Vossepoel, A model based method for retinal blood vessel detection. Computers in Biology and Medicine 34, 209 (2004).

35. T. Walter and J. C. Klein, Segmentation of color fundus images of the human retina: Detection of the optic disk and the vascular tree using morphological techniques, Second International Symposium on Medical Data Analysis (2005), Vol. 2199, pp. 282-287.

36. F. Zana and J. C. Klein, Segmentation of vessel-like patterns using mathematical morphology and curvature evaluation. IEEE Transactions on Image Processing 10, 1010 (2001). 